\section{SO5.2 ADOLESCENT CHLAMYDIA RATES ADJUSTED FOR SCREENING HETEROGENEITY}

Lizzi Torrone*. Centers for Disease Control and Prevention, Atlanta, USA

10.1136/sextrans-2015-052270.36

After chlamydia became a nationally notifiable condition in the United States, reported case rates increased steadily, likely a result of more complete reporting, expanded screening efforts, and use of more sensitive diagnostic tests. Reported case rates of chlamydia are highest among young women, a consequence of high prevalence in this population (as evidenced by estimates from national population-based surveys) and of targeted screening efforts (current guidelines in the United States recommend screening for all sexually active females aged $<25$ years). However, after a decade of increasing reported case rates, case rates have recently declined among adolescent women aged 15-19 years: during 2001-2011, case rates increased $49.8 \%$ (2,327 to 3,485 per 100,000 females) and then decreased $15.1 \%$ during 2011-2014 (3,485 to 2,960 per 100,000 females). We explored possible reasons for decreases in chlamydia case rates among adolescent females using available national data and highlight how decreases in screening coverage among key populations may be impacting reported case rate trends.

\section{S05.3 CALCULATING RISK AMONG MEN WHO HAVE SEX WITH MEN}

Kyle Bernstein*. Centers for Disease Control and Prevention, Atlanta, USA

\subsection{6/sextrans-2015-052270.37}

In the United States (US) in 2013, men who have sex with men (MSM) accounted for $75 \%$ of reported primary and secondary syphilis and over $80 \%$ of new HIV diagnoses. However, describing trends in MSM disparities are challenging. Few estimates of the size of the population of men who have sex with men (MSM) in the US exist. The National Survey of Family Growth (NSFG) is a probability sample of American men and women aged 15-44. We used the weighted proportion of males surveyed during the 2006-2010 cycle of NSFG who reported having ever had sex with men to estimate the population prevalence of MSM in the US; racial/ethnic and age specific were also developed. We estimated the population of MSM by applying the proportions of men who reported ever having sex with a man from NSFG to the 2010 census estimates of the number of men 15-44. Rates of primary and secondary (P\&S) syphilis among MSM were calculated by dividing the number of reported P\&S syphilis cases among MSM by the estimated US MSM population. Age and racial/ethnic rates were also estimated. Among the 10,403 males participating in the 2006-2010 NSFG cycle, the proportion who reported ever having sex with a man was $5.1 \%$; we estimated 3.2 million MSM aged 15-44 in the US. By applying metrics from a national probability sample of males, we were able to estimate conservative rates of P\&S syphilis among important sub-populations of MSM. However, numerous challenges exist to developing robust MSM population-based estimates of disease burden including how to determine the gender of sex partners on male case reports for syphilis, chlamydia, gonorrhoea, and HIV and the estimation of sub-population specific denominators (e.g. racial/ethnic groups) for MSM.
S05.4 POTENTIAL INCONGRUITIES IN RELATIVE MEASURES OF DISPARITIES IN SEXUALLY TRANSMITTED DISEASES

Harrell Chesson*. Centers for Disease Control and Prevention, Atlanta, USA

10.1136/sextrans-2015-052270.38

Sexually transmitted infections (STIs) in pregnancy prevalent in many resource limited settings are associated with adverse pregnancy outcomes that include spontaneous abortion, stillbirths, preterm rupture of membranes, premature delivery and postpartum endometritis.

Antenatal clinic attendance provides opportunity to screen the women for STIs, offer prompt treatment for curable STIs or prophylaxis for viral infections such as HIV and to promote prevention. Although routine screening for syphilis and HIV is recommended as part of antenatal care (ANC), optimal screening rates have not been achieved. This is due antenatal clinic nonattendance, non-availability of screening tests and where available, health system inefficiencies such long wait time before test results are available or need for women to make return visits to receive results. Use of point-of care tests that detect syphilis and HIV have been shown to increase the number of women tested for HIV and syphilis, are inexpensive, easy to use and do not require a sophisticated laboratory. In resource limited settings, prompt detection and treatment of the other STIs is hampered by lack of screening tests. Often, health providers rely on syndromic approach to diagnose and manage STIs. This approach alleviates the need for laboratory services but misses women with asymptomatic infections and has poor diagnostic performance. Pregnant women with STIs are at an increased risk of acquiring HIV and transmitting the infection to their infants. Increased availability of HIV testing and provision of effective PMTCT regimens has dramatically reduced the number of new paediatric HIV infections. However, STIs, apart from HIV, have not been viewed as a public health priority and adequate resources allocated to combat them. To optimise maternal and infant outcomes, there is need to prioritise development of low-cost tests to detect STIs during pregnancy and to ensure women are provided with effective treatment promptly.

\section{S05.5 NETWORK FORMATION AS A DETERMINANT OF SPATIAL DISPARITY}

Ian Spicknall ${ }^{*}$, HW Chesson, CG Patel, TL Gift, KT Bernstein, SO Aral. Centers for Disease Control and Prevention, Atlanta, USA

\subsection{6/sextrans-2015-052270.39}

Introduction Racial disparities in the burden of sexually transmitted disease have been documented and described for decades. Several disparity measures, such as the black-to-white rate ratio, the Index of Disparity (ID), and the Gini coefficient, have been used to quantify disparities in STDs. Although these measures are generally useful and informative, in some scenarios the results of these measures may seem incongruous with reasonable, practical assessments of disparity. The purpose of this study was to provide examples of such incongruities in the context of racial disparities in STDs.

Methods We examined a population consisting of 312.5 million people overall, with 200 million non-Hispanic Whites, 40 million non-Hispanic Blacks, 55 million Hispanics, 15 million Asian/Pacific Islanders, and 2.5 million American Indian/Alaskan Natives (AI/AN), similar to the actual US population. Case numbers of a hypothetical STD for each racial group were chosen 
arbitrarily (yet purposefully) to yield illustrations of potential incongruities among disparity measures.

Results We found several hypothetical examples of incongruities among disparity measures. For example, the ID was about ten times higher when all STD cases occurred among AI/AN than when all cases occurred among non-Hispanic Blacks. As another example, the ID indicated that disparity was less when all STD cases occurred among non-Hispanic Blacks than when each of the five racial groups accounted for one fifth of all STD cases. Conclusion Relative measures of racial disparity in STDs can be useful to illustrate the burden of disparity, to assess trends, and to inform the targeting of prevention resources. However, in some scenarios the disparity measures can be incongruous with reasonable, practical assessments of disparity, such as when the ID is biased against non-Hispanic Blacks. The ID is more prone to these incongruities than measures which account for population size, such as the Gini coefficient or the weighted ID.

Disclosure of interest statement The authors have no conflicts to declare. No pharmaceutical grants were received in the development of this study.

\section{S06 - STI vaccines: Advancing the global agenda}

\section{S06.1 THE GLOBAL ROADMAP FOR STI VACCINE DEVELOPMENT: MOVING FORWARD}

Sami Gottlieb*. World Health Organization, Geneva, Switzerland

\subsection{6/sextrans-2015-052270.40}

The global STI vaccine roadmap outlines critical next steps to accelerate development of new STI vaccines according to nine priority action areas: 1) obtaining better epidemiologic data; 2) improving the understanding of STI natural history and the burden of sequelae; 3) modelling the theoretical impact of STI vaccines; 4) advancing basic science research for STI vaccines; 5) conducting basic and translational studies in human clinical settings as soon as possible; 6) defining preferred product characteristics for 1 st generation vaccines; 7) expediting clinical development and evaluation; 8) planning for vaccine introduction in advance; and 9) encouraging investment in STI vaccine development. This presentation will review the global roadmap for STI vaccine development, discuss key ongoing activities to implement the roadmap and advance the global STI vaccine agenda, and important next steps to continue to catalyse STI vaccine development.

\section{S06.2 HERPES SIMPLEX VIRUS VACCINE DEVELOPMENT: PIPELINES AND POSSIBILITIES}

Christine Johnston*. University of Washington, Seattle, USA

\subsection{6/sextrans-2015-052270.41}

Genital herpes simplex virus (HSV) infection causes recurrent genital ulcers, neonatal herpes, and increases the risk of HIV acquisition. HSV-2, the most common cause of genital herpes, is highly prevalent worldwide, with an estimated 417 million people infected between the ages of 15-49. The urgent need for a prophylactic vaccine against genital HSV has been long recognized. Multiple glycoprotein subunit vaccines candidates have been tested but none have successfully prevented HSV-2 genital ulcer disease in phase III trials. Despite these findings, there is strong interest in pursuing novel vaccine platforms to induce immune responses to protect against HSV acquisition. Therapeutic vaccines to reduce genital symptoms and viral shedding in persons already infected with HSV-2 are also being tested in early phase clinical trials. The global STI vaccine roadmap provided a framework for research priorities to move the HSV vaccine field forward. This presentation will review 1) lessons from prior clinical trials of HSV vaccines, 2) new insights into immunology of HSV infection, 3) current status of HSV vaccine pipeline, with an emphasis on candidates currently in clinical trials and 4) discussion of clinical trial design issues unique to HSV.

\section{S06.3 CHLAMYDIA TRACHOMATIS VACCINE DEVELOPMENT: NEW TOOLS BRING NEW HOPE}

Peter Timms*. University of Sunshine Coast, Maroochydore, Australia and Queensland University of Technology, Brisbane, Australia

\subsection{6/sextrans-2015-052270.42}

Despite decades of research, progress towards a vaccine for genital Chlamydia trachomatis infection and disease has been slow, with only modest levels of protection being achieved to date. In the last three years in particular, there have been several significant advances that give renewed optimism for an effective vaccine. In 2014, the WHO published a Road Map for STI vaccine development, listing a range of key objectives that need to be addressed. This presentation will discuss several of these objectives and the recent progress being made, including (a) understanding the relationship between pathogen genomes and disease severity, (b) use of non-mouse models for evaluating vaccines, (c) better understanding of disease pathogenesis using a rapidly expanding genetic toolbox, (d) recent promising vaccine trials.

\section{S06.4 APPROACHING THE APEX: TECHNOLOGY INNOVATIONS FACILITATING THE DEVELOPMENT OF A GONOCOCCAL VACCINE}

Scott D Gray-Owen*. Department of Molecular Genetics, University of Toronto, Toronto, Ontario, Canada

\subsection{6/sextrans-2015-052270.43}

Despite remaining as one of the leading causes of sexually transmitted infections, with sequelae ranging from ectopic pregnancy and infertility caused by scarring of the reproductive tract to blindness of children born to infected mothers, Neisseria gonorrhoeae has become a forgotten plague. This status has begun to change as multidrug resistant strains of $N$. gonorrhoeae have emerged, raising the frightening prospect of untreatable infections. Fortunately, as a renewed sense of urgency for the development of a prophylactic gonococcal vaccine has arisen, there have been several great strides made that will facilitate the development of a gonococcal vaccine. First, a new appreciation of the value of phenotypic and genome-based analyses of $N$. gonorrhoeae variants has led to increased recovery and characterization of bacteria from clinical specimens rather than simple PCRbased molecular diagnosis. Second, new insights regarding immune responses that facilitate N. gonorrhoeae persistence suggest immunological correlates that might afford protective memory. Third, the development of vaginal and transcervical uterine 